\title{
Association of Vitiligo with Other Autoimmune Disorders
}

Khawer Saleem* and Waqar Azim

Department of Dermatology, Al-Ahalia Hospital, Al-Ain, UAE

*Corresponding author: Khawer Saleem, Consultant Dermatologist, Al-Ahalia Hospital, Al-Ain, UAE, Tel: 00971-3-7555997; E-mail: dr_khawer_saleem@yahoo.com Received date: May 03, 2016; Accepted date: May 05, 2016; Published date: Oct 25, 2016

Copyright: (C) 2016 Saleem K, et al. This is an open-access article distributed under the terms of the creative commons attribution License, which permits unrestricted use, distribution, and reproduction in any medium, provided the original author and source are credited.

\begin{abstract}
Objective: To assess the association of Vitiligo with other autoimmune disorders and to see the familial association.

Design: Prospective case control study.

Duration and place of study: Jan 2005 to Jan 2007. Skin department PNS Shifa Hospital Karachi.

Material and methods: Hundred cases of vitiligo of all ages and both genders were included in the study. Diagnosis of Vitiligo was done by clinical examination and was confirmed by Wood's lamp examination. Skin biopsy was carried out for standard pathologic examination to help confirm the diagnosis in two cases. The presence of vitiligo in parents and siblings was probed and was recorded. History was taken and physical examination was done to see any evidence of presence of following six autoimmune disorders, alopecia areata, diabetes mellitus, thyroid disorders (both hyper and hypothyroidism), Addison disease, systemic lupus erythematosus and Rheumatoid arthritis. The selected patients were subjected to following tests, blood complete picture, blood glucose levels random and fasting, thyroid function tests (T3, T4 and TSH levels), serum cortisol levels at 0800 hrs. ANA and RA factor in cases of suspected rheumatoid arthritis cases. Fifty cases of chronic eczema reporting in skin outpatient department were taken as control. The control subjects were also studied for the presence of above mentioned autoimmune disorders and the above mentioned laboratory tests were carried out.
\end{abstract}

Results: We analyzed 100 cases of vitiligo, 38 were males and 62 females. The mean age of presentation was $32 \pm 17.6$ yrs. Positive family history was found in $25(25 \%)$ subjects. Twenty $(20 \%)$ cases had evidence of presence of other autoimmune disorders. Eight (8\%) patients had alopecia areata, 2(2\%) patients had insulin dependent diabetes mellitus, $2(2 \%)$ patients had RA positive Rheumatoid arthritis, $8(8 \%)$ patients had thyroid disorders (5 had hyperthyroidism and 3 had hypothyroidism). In the control group of 50 cases of chronic eczema, 3(6\%) cases had the evidence of presence of autoimmune disorders, $2(4 \%)$ cases had alopecia areata and $1(2 \%)$ case had hyperthyroidism.

Conclusion: Vitiligo is strongly associated with other autoimmune disorders and has positive family history. Patients having vitiligo should be looked for other autoimmune disorders especially the thyroid disorders.

Keywords: Vitiligo; Autoimmune disorders; Eczema

\section{Introduction}

Vitiligo is an acquired skin disorder caused by the destruction of melanocytes in the epidermis resulting in well-defined white patches which are often symmetrically distributed. It occurs with an estimated worldwide incidence of $0.5 \%$ to $4 \%$, with equal frequency in males and females. The incidence of vitiligo in those with racially pigmented skin is higher and the social impact is greater [1]. About $6 \%$ to $38 \%$ of patients have positive family history indicating a hereditary factor However, the inheritance pattern of the disorder is consistent with that of a polygenic trait and not just by simple Mendelian transmission. Vitiligo can develop at any age, however in $50 \%$ of the cases it develops before the age of 20 yrs [2]. Amelanocytic macules in vitiligo are found particularly in areas that are normally hyper pigmented and at areas subjected to repeated trauma and friction. The distribution of the lesions is usually symmetrical, although sometimes it is unilateral and has dermatomal arrangement [3]. Vitiligo is a multifactorial disorder in which multiple genes contribute to the expression of the phenotype. Genes which are associated with vitiligo recently are, an SMOC2 variant (rs13208776) and NACHT-leucine-rich-repeat protein-1 (NALP1) [4]. Vitiligo is characterized by progressive de-pigmentation of the skin due to autoimmune destruction of epidermal melanocytes and/or their auto-destruction due to toxic melanin biosynthesis metabolites [5]. ROS (reactive oxygen species) and $\mathrm{H}_{2} \mathrm{O}_{2}$ in excess can damage biological processes of malanogenesis [6]. The autoimmune theory proposes alteration in humoral and cellular immunity in the destruction of melanocytes. Multiple autoimmune syndrome (MAS) is a newly defined association of immunological disorders. It's classified as the coexistence of three or more autoimmune diseases, one of which is a dermatological autoimmune disease such as vitiligo psoriasis or scleroderma. The MAS represent the best example of polyautoimmunity as well as the effect of a single genotype on diverse phenotypes [7].

Thyroid disorders, particularly Hashimoto thyroiditis and Graves' disease, other endocrinopathies, such as Addison disease, diabetes 
mellitus, alopecia areata, pernicious anemia, inflammatory bowel disease, psoriasis, and autoimmune poly-glandular syndrome are all associated with vitiligo [8]. The most convincing evidence of an autoimmune pathogenesis is the presence of circulating antibodies in patients with vitiligo. The role of humoral immunity is further supported by the observation that melanocytes are destroyed in healthy skin engrafted onto nude mice injected with vitiligo patient sera $[9,10]$. In addition to the involvement of humoral immune mechanisms in the pathogenesis of vitiligo, strong evidence indicates involvement of cellular immunity in vitiligo. Destruction of melanocytes may be directly mediated by auto reactive CD8+ T-cells. Activated CD8+ T-cells have been demonstrated in peri-lesioned vitiligo skin [11]. In addition, melanocyte-specific $\mathrm{T}$ cells have been detected in peripheral blood of patients with autoimmune vitiligo [12].

In this study we aimed to assess the association of vitiligo with six other autoimmune disorders namely alopecia areata, diabetes mellitus, thyroid disorders, Addison disease, Rheumatoid arthritis and systemic lupus erythematosus in our local population.

\section{Material and Methods}

This was a cross sectional prospective study conducted in the out patients' department of dermatology, PNS Shifa Hospital Karachi, Pakistan from January 2005 to January 2007. The study group included 100 new cases of various types of vitiligo attending the dermatology department. Selection was done on the basis of random sequential sampling. Patients of all ages and both genders were included in the study. Diagnosis of vitiligo was done by clinical examination and was confirmed by Wood's lamp examination. Skin biopsy was carried out for standard pathologic examination to help confirm the diagnosis in dubious cases. Fifty cases of chronic eczema reporting in out patients' department were taken as control. Informed consent, from all patients in subject and control groups, was obtained prior to the study. History of presence of vitiligo in parents and siblings was sought and recorded. The total duration of occurrence of vitiligo and the clinical pattern of its presentation were noted and recorded. History was taken and physical examination was done in subject and control groups to see the evidence of following six autoimmune disorders, alopecia areata, diabetes mellitus, thyroid disorders (both hyper and hypothyroidism), Addison disease, systemic lupus erythematosus and Rheumatoid arthritis. Following investigations were carried out in subject and control groups, complete blood picture(CBC), blood sugar levels random and fasting, thyroid function tests (T3, T4 and TSH levels), anti-thyroid antibodies, serum cortisol level at $0800 \mathrm{hrs}$. anti-nuclear factor (ANA) and rheumatoid arthritis factor (RA factor) in cases of suspected rheumatoid arthritis cases.

The collected data was analyzed using SPSS version 11 software. Demographic data such as age, gender, duration of illness and other pieces of information were collected with a checklist structured Performa after the patients' consent. Chi-square test was used to calculate the ' $\mathrm{p}$ ' value. The $\mathrm{p}<0.05$ was considered as significant.

\section{Results}

We analyzed 100 cases of vitiligo. There were 38 (38\%) male and $62(62 \%)$ female patients with a ratio of 1:1 [6]. The mean age of presentation was $32 \pm 17.6$ yrs. Twenty-eight (28\%) subjects had vitiligo of 01 years or less of duration. Forty-eight (48\%) patients had vitiligo of 1 to 5 years of standing. Ten (10\%) subjects had vitiligo of more than 10 years of duration (Table 1). Vulgaris type of vitiligo was the commonest variety, seen in fifty-two (52\%) subjects followed by focal type seen in 24(24\%) subjects (Table 2). 25 (25\%) subject's vitiligo was found in either parents or siblings. $20(20 \%)$ cases had evidence of presence of other autoimmune disorders. In eight (8\%) patient's alopecia areata was found, 2 (2\%) patients had insulin dependent diabetes mellitus (type I), 2 (2\%) patients had RA factor positive rheumatoid arthritis, $8(8 \%)$ patients had thyroid disorders. Five of these 8 thyroid disorder cases had hyperthyroidism and 3 cases had hypothyroidism. No patient had clinical or biochemical evidence of Addison's disease or systemic lupus erythematosus (Table 3). Where as in the control group of chronic eczema patients, out of 50 cases, $3(6 \%)$ cases had the evidence of presence of other autoimmune disorders. $2(4 \%)$ cases had alopecia areata and $1(1 \%)$ case had hyperthyroidism. The $\mathrm{p}$ value less than 0.05 was taken as significant.

\begin{tabular}{|c|c|c|c|c|c|}
\hline $\begin{array}{l}\text { Age of } \\
\text { onset }\end{array}$ & $\begin{array}{l}\text { Number of } \\
\text { patients }\end{array}$ & $\begin{array}{l}\text { Duration of } \\
\text { Vitiligo }\end{array}$ & $\begin{array}{l}\text { Number of } \\
\text { patients }\end{array}$ & Gender & $\begin{array}{l}\text { Percenta } \\
\text { ge }\end{array}$ \\
\hline$<20$ yrs. & $26(26 \%)$ & $<1 \mathrm{yrs}$. & $28(28 \%)$ & & \\
\hline $20-40$ yrs. & $38(38 \%)$ & $1-5 \mathrm{yrs}$ & $48(48 \%)$ & Males & $38.00 \%$ \\
\hline $40-60$ yrs. & $22(22 \%)$ & 5-10 yrs. & $14(14 \%)$ & & \\
\hline$>60 \mathrm{yrs}$ & $4(4 \%)$ & $>10$ yrs & $10(10 \%)$ & Females & $62.00 \%$ \\
\hline
\end{tabular}

Table 1: Demographics of 100 patients of vitiligo.

\begin{tabular}{|l|l|l|}
\hline Pattern of Vitiligo & Frequency & Percentage \\
\hline Generalized (vulgaris) & 52 & $52.00 \%$ \\
\hline Focal & 24 & $24.00 \%$ \\
\hline Acrofacial & 16 & $16.00 \%$ \\
\hline Segmental & 4 & $4.00 \%$ \\
\hline Flextural & 4 & $4.00 \%$ \\
\hline
\end{tabular}

Table 2: Frequency of different patterns of vitiligo $(n=100, n$-total number).

\begin{tabular}{|l|l|l|l|}
\hline Autoimmune disorders & $\begin{array}{l}\text { Frequency of } \\
\text { association } \\
\text { in subjects }(\mathbf{n = 1 0 0 )}\end{array}$ & $\begin{array}{l}\text { Frequency of } \\
\text { association in } \\
\text { controls } \\
(\mathbf{n}=50)\end{array}$ & p-value \\
\hline Alopecia areata & $8(8 \%)$ & $2(4 \%)$ & $p<0.03 \mathrm{~S}$ \\
\hline Hyperthyroidism & $5(5 \%)$ & $1(2 \%)$ & $p<0.02 \mathrm{~S}$ \\
\hline Hypothyroidism & $3(3 \%)$ & Nil & p<0.03 S \\
\hline Type I diabetes mellitis & $2(2 \%)$ & Nil & p<0.02 S \\
\hline Rheumatoid arthritis & $2(2 \%)$ & Nil & p<0.02 S \\
\hline Addison disease & Nil & Nil & - \\
\hline SLE & Nil & Nil & - \\
\hline S Significant; & &
\end{tabular}

S-Significant; n-Total number

Table 3: Autoimmune disorders associated with vitiligo. 


\section{Discussion}

Vitiligo is characterized by progressive skin de-pigmentation due to autoimmune destruction of epidermal melanocytes. As suggested for other autoimmune diseases, vitiligo susceptibility may involve both target organ-specific genes and immune response genes [13]. The autoimmune theory proposes alteration in humoral and cellular immunity in the destruction of melanocytes of vitiligo [9]. Histological evidence further supports an autoimmune etiology. Vitiligo lesions have an infiltrate of inflammatory cells, particularly cytotoxic and helper T-cells and macrophages [14].

The female preponderance in our study $1.6: 1$ is in accordance with certain other studies in this regard which confirm female preponderance. The higher frequency of Vitiligo in females is in consistence with certain other studies in this regard [15]. We found the frequency of associated autoimmune disorders significantly higher in patients with vitiligo than in control group in our study. The frequency of association was $20 \%$ in vitiligo patients as compared to $6 \%$ in the control group, the ' $p$ ' value was less than 0.03 , which being significant. The association was highest with thyroid disorders (8\%). This association was more with hyperthyroidism. The association between vitiligo and autoimmune thyroiditis (Hashimoto's disease) is well documented in adults and children [16]. The association found with thyroid disorders was less in our study as compared to another study in which the association with autoimmune thyroiditis in adults had been around $30 \%$, amongst the vitiligo patients [17]. The other autoimmune disorders associated with vitiligo in our study were alopecia areata, diabetes mellitus, and Rheumatoid arthritis. These findings are similar to certain similar studies that analyzed the association between vitiligo and other autoimmune diseases [18]. These associations are indicative of the fact that vitiligo shares a common genetic etiologic links with these other autoimmune disorders. The immune system is undeniably involved in the pathogenesis, as evidenced by the effectiveness of immuno-modulatory agents, such as corticosteroids and calcineurin inhibitors in the treatment of vitiligo [19].

In our study we obtained a history of presence of vitiligo in parents and siblings in $20 \%$ of the cases which is similar to such other studies in this regard. Some studies have shown frequency of vitiligo in probands' siblings about 18 times the population frequency, suggesting a major genetic component in disease pathogenesis [20]. One study suggests that one fifth of other family members also have vitiligo [21]. Our study also supports genetic as well as non-genetic autoimmune component in the etiology of vitiligo.

\section{Conclusion}

There is strong association of vitiligo with other autoimmune disorders and has a positive family history indicating that both genetic as well as non-genetic autoimmune component in the etiology of vitiligo. Patients having vitiligo should be looked for other autoimmune disorders especially the thyroid disorders.

\section{Conflict of Interest}

No conflict of interest declared.

\section{References}

1. Halder R, Taliaferro S. Vitiligo. In: Wolff K, Goldsmith L, Katz S, Gilchrest B, Paller A, Lefell D (Eds). Fitzpatrick's Dermatology in General Medicine (7thedn). New York, NY: McGraw-Hill; 2008:72.

2. Habif TP. Light-related diseases and disorders of pigmentation. In: Habif TP (Ed.) Clinical dermatology (5thedn.) Philadelphia. 2009.

3. Viles J, Monte D, Gawkrodger DJ (2010) Vitiligo. BMJ. 341: c3780.

4. Al-Dain Marzouka N (2010) SMOC2 gene variant and the risk of vitiligo in Jordanian Arabs. Eur J Dermatol 20: 701-704.

5. Ortonne J (2008) Vitiligo and other disorders of hypopigmentation. Dermatology 1: 65.

6. Glassman SJ (2011) Vitiligo, reactive oxygen species and T-cells. Clin Sci (Lond) 120: 99-120.

7. Anaya JM, Adriana JC, Ricardo RV, Roger PT, Levy A (2012) The Multiple Autoimmune Syndromes. A Clue for the Autoimmune Tautology. Clin Rev Allergy Immunol 43: 256-264.

8. Matz H, Tur E (2007) Vitiligo. Curr Probl Dermatol. 35: 78-102.

9. Rashtak S, Pittelkow MR (2008) Skin involvement in systemic autoimmune diseases. Curr Dir Autoimmun 10: 344-358.

10. Arican O, Kurutas EB (2008) Oxidative stress in the blood of patients with active localized vitiligo. Acta Dermatovenerol Alp Panonica Adriat 17: 12-16.

11. Poole ICL, Luiten RM (2008) Autoimmune etiology of generalized vitiligo. Curr Dir Autoimmun 10: 227-243.

12. Wankowicz-Kalinska A, van den Wijngaard RM, Tigges BJ, Westerhof W, Ogg GS, et al. (2003) Immunopolarization of CD4+ and CD8+ T cells to Type-1-like is associated with melanocyte loss in human vitiligo. Lab Invest 83: 683-695.

13. Spritz RA (2008) The genetics of generalized vitiligo. Curr Dir Autoimmun 10: 244-257.

14. Oiso NT, Fukai K, Kabashima K, Kawada A, Suzuki T (2011) Generalized vitiligo and associated autoimmune diseases in japanese patients and their families. Allergol Int 60(4): 505 -508.

15. Zum B, Alam M, Khondker L, Siddiqua A, Alam MN, et al. (2013) Association of other Autoimmune Diseases in Vitiligo Patients. Community Based Medical Journal 02: 57-61.

16. Kakourou T, Kanaka-Gantenbein C, Papadopoulou A, Kaloumenou E, Chrousos GP (2005) Increased Prevalence of Chronic Autoimmune (Hashimoto's) Thyroiditis in Children and Adolescents with Vitiligo. J Am Acad Dermatol 53(2): 220-223.

17. Yang $\mathrm{Y}$, Lin X, Fu W, Luo X, Kang K (2010) An approach to the correlation between vitiligo and autoimmune thyroiditis in Chinese children. Clin Exp Dermatol 35: 706-710.

18. Rashtak S, Pittelkow MR (2008) Skin involvement in systemic autoimmune diseases. Curr Dir Autoimmun 10: 344-358.

19. Lotti T, Gori A, Zanieri F, Colucci R, Moretti S (2008) Vitiligo: new and emerging treatments. Dermatol Ther 21: 110-117.

20. Pajvani U, Ahmad N, Wiley A (2006) The relationship between family medical history and childhood vitiligo. J Am Acad Dermato 55: 238-244.

21. Silverberg NB (2010) Update on childhood vitiligo. Curr Opin Pediatr 22: $445-452$ 